\title{
An infant with glutaric aciduria type IIc diagnosed with a novel mutation
}

\author{
Sedat Işıkay¹, Ayhan Yaman², Serdar Ceylaner ${ }^{3}$ \\ ${ }^{1}$ Division of Pediatric Neurology, Department of Pediatrics, Hasan Kalyoncu University, Faculty of Medicine, ${ }^{2}$ Division of \\ Pediatric Intensive Care Unit, Department of Pediatrics, Gaziantep University Faculty of Medicine, Gaziantep, ${ }^{3}$ Intergen \\ Genetic Center, Ankara, Turkey. E-mail: dr.sedatisikay@mynet.com \\ Received: 28th November 2016, Revised: 19th January 2017, Accepted: 19th January 2017
}

\begin{abstract}
SUMMARY: Işıkay S, Yaman A, Ceylaner S. An infant with glutaric aciduria type IIc diagnosed with a novel mutation. Turk J Pediatr 2017; 59: 315-317.

Glutaric aciduria type II is a rare inborn error of metabolism. The clinical picture is highly variable with symptoms ranging from acute metabolic decompensations to chronic, mainly muscular problems or even asymptomatic cases. Herein we described a 7-month-old female patient presented with respiratory failure and diagnosed with glutaric aciduria type II via whole exome sequencing that exhibited one known and a novel mutation. Her blood and urine analyses were all normal. After the diagnosis, dramatic and sustained improvement on a low-fat, low-protein, and high-carbohydrate diet supplemented with oral riboflavin and carnitine was determined. In especially hypotonic patients with unknown etiologies, though the blood and urine analyses are normal, glutaric aciduria type II should also be kept in mind and genetic tests may be required for the diagnosis.
\end{abstract}

Key words: glutaric aciduria, genetic test, child.

Glutaric aciduria type II (GA II) is an autosomal recessive disorder of fatty acid, amino acid, and choline metabolism. Excretion in large amounts of not only of glutaric acid, but also of lactic, ethylmalonic, butyric, isobutyric, 2-methylbutyric, and isovaleric acid is the characteristic of GA II that differentiates it from Glutaric aciduria type $\mathrm{I}^{1}$. Mutations in 3 main genes, the alpha (ETFA) and beta (ETFB) subunits of electron transfer flavoprotein, and electron transfer flavoprotein dehydrogenase (ETFDH) have been determined to be responsible from the GA $\mathrm{II}^{2,3}$. The clinical picture and disease onset may vary depending on the location and nature of the mutations ${ }^{1-5}$.

Herein we will describe a case presented with respiratory failure and diagnosed with GA II via Whole Exome Sequencing analysis that exhibited a novel mutation.

\section{Case Report}

A 7-month-old female was admitted to the intensive care unit (ICU) with weakness, hypotonia and dyspnea. Her parents expressed that, she had a progressive respiratory failure in last week. Although everything was normal in first 4 months of her life, she was defined as hypotonic later. She was the first and the only child of non-consanguineous parents. In ICU, she was intubated and placed on mechanical ventilator. Her physical examination revealed axial hypotonia without the presence of deep tendon reflexes. However, although she was intubated, her eye-follow was good. Her eye and hearing examinations were normal. In laboratory examinations; her hemogram, liver and kidney function tests, creatine kinase, uric acid, ammonia, and lactate levels and blood gas analysis, together with blood carnitine and acylcarnitine analysis and urine organic acid analysis, blood and urine aminoacid levels were all normal. Her spinal muscular atrophy (SMA) gene analysis was normal. Brain magnetic resonance imaging was unremarkable. As empirical treatment, L-carnitin $100 \mathrm{mg} /$ $\mathrm{kg} /$ day and multiple vitamins were started. She was followed on mechanical ventilation for 1 month and then extubated. Then, the patient displayed progressive recovery especially with the support of physiotherapy. Her parents regret muscle biopsy. For the diagnosis, genetic analysis was performed by whole exome sequencing (WES) technology 
(Nextseq-Illumina) and compound heterozygote NM_004453.3 (ETFDH): p.L377P (c.1130T >C)/ p.H401qQfs*3) (c.1198_1201delACTC) variations were detected. P.L377P variation was described as a disease-causing variant in HGMD-Public database (CM073029). The other variation was a novel mutation but as it causes frame shift and a premature stop codon, it is most probably also a disease-causing variant. In silico analyses both variants were presented as damaging variants and that is why we reported these variants as pathogenic.

The patient was diagnosed with glutaric aciduria type IIc and riboflavin $100 \mathrm{mg} /$ day treatment was started. The patient is now 15 months old and she can crawl and walk after standing by a support. She can talk with words having 2 syllables such as "mummy," and "daddy." Informed consent was received from the family.

\section{Discussion}

In this case report, we described a rare cause of acute respiratory failure and hypotonia, in an infant with glutaric aciduria type IIc. Glutaric aciduria II or multiple acyl-CoA dehydrogenase deficiency is an autosomal recessive disorder affecting fatty acid, aminoacid, and choline metabolism ${ }^{1-3}$. The disease is characterized by recurrent episodes of vomiting, lethargy, hypoglycemia, and metabolic acidosis. Muscle pain, weakness, and lipid-storage myopathy are the main presentations of muscle involvement in GA-II2,5,6. Three different clinical forms with different ages of onset are described. Type I is the lethal form with neonatal-onset and accompanying congenital anomalies. Generally non-ketotic hypoglycemia, metabolic acidosis, excretion of large amounts of fatty acid, and amino acid metabolites accompany the lethal form. Type II is again a neonatal-onset form but without congenital anomalies while type III is the late onset form ${ }^{5,7}$.

Urine organic acid analysis may not be helpful in the stable form of GA II, because levels are often normal except during a symptomatic period. During acute episodes, the analysis may demonstrate dicarboxylic acid compounds (suberic acid, sebacic acid, and adipic acid). Acylcarnitine analysis with tandem mass spectrometry is a very useful diagnostic tool for GA II. Acylcarnitine profiling by tandem mass spectrometry screening of serum or dried blood spot samples characteristically shows increased concentrations of short-, medium-, and longchain acylcarnitines. However, in the milder form, the abnormal urinary organic aciduria may only be determined during episodes of stress or exacerbations ${ }^{1-4,7}$. Interestingly, both of urine organic acids and acyl carnitine profile were normal in our patient. Therefore, we decided to obtain whole exome sequencing analysis in the patient.

The mutations in at least 3 different genes involved in electron transfer in the mitochondrial respiratory chain: ETFA, ETFB, and ETFDH were described as the etiology of GTA II. Although, any differences in the clinical phenotypes were not reported regarding the effected gene, ETFA, ETFB, or ETFDH meaning that all clinical forms can be caused by defects in any of these three genes; GTA II is sub-grouped as glutaric aciduria IIa, IIb, and IIc according to the defects in these 3 genes, respectively ${ }^{1-4}$. In our patient, two different mutations in ETFDH gene were determined.

Previously, different mutations in ETFDH gene with different clinical pictures were reported $^{8,9}$. In 2007, Gempel et al. ${ }^{10}$ reported that, homozygous or compound heterozygous mutations in the ETFDH gene can result in isolated myopathy with secondary coenzyme Q10 deficiency and they suggested that, if both urine organic acids and blood acyl-carnitine analysis are normal, genetic counseling would be offered for further mutational analysis of ETFDH ${ }^{9}$. In our case, similarly only the WES analysis confirmed the diagnosis with p.L377P (c.1130T >C)/p.H401qQfs*3) (c.1198_1201delACTC) (compound heterozygous) mutation in ETFDH. This is a new mutation for GA IIc. Oral riboflavin was reported consistently to improve the conditions of patients with glutaric aciduria type II dramatically, with almost complete resolution of symptoms and signs in milder cases as in our case ${ }^{11,12}$. Dramatic and sustained improvement on a low-fat, low-protein, and high-carbohydrate diet supplemented with oral riboflavin and carnitine was determined in our patient.

In conclusion, we have reported a very rare presentation of GA IIc in a patient with respiratory failure. In especially hypotonic patients with unknown etiologies, though the 
blood and urine analyses are normal, GA IIc should also be kept in mind and genetic tests may be required for the diagnosis.

\section{REFERENCES}

1. Olsen RK, Olpin SE, Andresen BS, et al. ETF-DH mutations as a major cause of riboflavin-responsive multiple Acyl-CoA dehydrogenation deficiency. Brain 2007; 130: 2045-2054.

2. Yamada K, Kobayashi H, Bo R, et al. Clinical, biochemical and molecular investigation of adult-onset glutaric acidemia type II: Characteristics in comparison with pediatric cases. Brain Dev 2016; 38: 293-301.

3. Freneaux E, Sheffield VC, Molin L, Shires A, Rhead WJ. Glutaric acidemia type II. Heterogeneity in beta-oxidation flux, polypeptide synthesis, and complementary DNA mutations in the alpha subunit of electron transfer flavoprotein in eight patients. J Clin Invest 1992; 90: 1679-1686.

4. Colombo I, Finocchiaro G, Garavaglia B, Garbuglio N, Yamaguchi S, Frerman FE, Berra B, DiDonato S. Mutations and polymorphisms of the gene encoding the beta-subunit of the electron transfer flavoprotein in three patients with glutaric acidemia type II. Hum Mol Genet 1994; 3: 429-435.

5. Loehr JP, Goodman SI, Frerman FE. Glutaric acidemia type II: heterogeneity of clinical and biochemical phenotypes. Pediatr Res 1990; 27: 311-315.
6. Prasad M, Hussain S. Glutaric aciduria type II presenting as myopathy and rhabdomyolysis in a teenager. J Child Neurol 2015; 30: 96-99.

7. Liang WC, Nishino I. State of the art in muscle lipid diseases. Acta Myol 2010; 29: 351-356.

8. Pollard LM, Williams NR, Espinoza L, et al. Diagnosis, treatment, and long-term outcomes of late-onset (type III) multiple acyl-CoA dehydrogenase deficiency. J Child Neurol 2010; 25: 954-960.

9. Beard SE, Goodman SI, Bemelen K, Frerman FE. Characterization of a mutation that abolishes quinone reduction by electron transfer flavoprotein-ubiquinone oxidoreductase. Hum Mol Genet 1995; 4: 157-161.

10. Gempel K, Topaloglu H, Talim B, et al. The myopathic form of coenzyme Q10 deficiency is caused by mutations in the electron-transferring-flavoprotein dehydrogenase (ETFDH) gene. Brain 2007; 130: 20372044.

11. Di Giacinto I, Buda S, Diamanti M, Tonon C, Pigna A, Melotti RM. Multiple acyl-coenzyme A dehydrogenase deficiency: diagnosis in adulthood, intensive care management and sequelae. Minerva Anestesiol 2014; 80: $1145-1146$

12. Cotelli MS, Vielmi V, Rimoldi M, et al. Riboflavinresponsive multiple acyl-CoA dehydrogenase deficiency with unknown genetic defect. Neurol Sci 2012; 33: 1383-1387. 